\title{
ETUDE COMPARATIVE DE LA DISTOMATOSE PANCRÉATIQUE CHEZ L'HOMME ET LES BOVIDÉS
}

\author{
Par H. GALliARD et P. GUYET-ROUSSET
}

La distomatose pancréatique n'existe pratiquement jamais en tant qu'infestation parasitaire isolée. Chez l'homme, dans les quelques cas rapportés, les auteurs la considèrent comme une complication éventuelle d'une distomatose hépato-biliaire intense. Chez les Bovidés elle est purement pancréatique et s'accompagne également d'une atteinte loco-régionale plus ou moins importante. Chez l'homme les signes d'insuffisance pancréatique sont précoces, rapidement très graves, et l'évolution se fait brièvement vers la mort, dans un tableau de cachexie extrême. Chez le bœuf, au contraire, le parasitisme semble remarquablement bien toléré et n'est souvent qu'une découverte de contrôle vétérinaire d'abattage.

Sur le plan parasitologique Clonorchis sinensis et Eurytrema pancreaticum diffèrent par certains caractères morphologiques et par leur biologie ; mais leur taille est semblable et leur comportement physiologique dans le pancréas, leur métabolisme sont comparables a priori.

Pourquoi une telle différence de retentissement organique devant une infestation parasitaire dont l'intensité, pour le moins, est souvent comparable ? C'est ce que nous avons cherché à expliquer par cette étude comparative.

\section{A) Eurytrema pancreaticum chez les Bovidés}

Nous avons réuni onze observations de distomatose bovine à E. pancreaticum provenant des abattoirs de Hanoï.

L'infection purement pancréatique est extrêmement commune et précoce chez le buffle au Tonkin. L'un de nous, avec D. V. Ngu (1941), a décrit une espèce un peu différente, E. tonkinense. Sa taille était plus grande, mais le ròle pathogène était probablement le même.

Au Brésil, Parreiras Horta (1918) signale la fréquence de l'infection à E. cœlomaticum, même chez les jeunes. C. Burle de Figueiredo (1928), puis Cesar Pinto et J. L. de Almeida (1935), décrivent le processus de pancréatite chronique que ce parasite provoque : prolifération du tissu conjonctif, destruction des acini glandulaires, 
conservation des îlots de Langherans. Burgraff (1933) étudie les lésions dues à $E$. pancreaticum provenant du Japon : dilatation des canaux pancréatiques, prolifération de la muqueuse et hyperplasie du tissu glandulaire, foyers nécrotiques centrés par des œufs. Magarinos Torres et Cesar Pinto (1936), dans le cas d'E. coelomaticum chez les Bovidés, décrivent en plus des granulomes avec cellules épithélioïdes en palissade, se développant à la surface de l'épithélium canalaire. Le tissu conjonctif néoformé est plutôt interlobulaire, mais parfois interacineux. Mais, surtout, ils observent une énorme quantité d'œufs situés dans la paroi même des canaux, pénétrant, par compression peut-être, dans le parenchyme où ils provcquent une infiltration lymphocytaire, la formation de cellules géantes et de la sclérose.

\section{B) Distomatose pancréatique humaine à Clonorchis sinensis}

La distomatose pancréatique doit être fréquente. Elle a été observée au Japon par Katsurada (1881-1898), dans 11,8 \% des cas par Ynoye (1903), signalée aussi par Yoshida (1931). Low, en 1909, en a décrit un cas. Rindfleisch, Askanazy, Nencioni ont observé des cas de distomatose pancréatique à Opisthorchis felineus. Newham a décrit les lésions anatomo-pathologiques d'un pancréas infesté de provenance inconnue.

Bien que la distomatose soit très commune au Tonkin, Gaide n'avait jamais trouvé de douves dans le pancréas. Sambuc, puis Sambuc et Beaujean en ont signalé deux cas la même année (1913).

En 1936, l'un de nous avait signalé deux cas de distomatose pancréatique. Ces deux cas étaient les troisième et quatrième observés chez des Tonkinois. Une étude histopathologique de ces deux cas a été publiée en 1938 (H. Galliard, Dang Van Ngu, Phan Huy Quat). Voici le résumé des deux observations cliniques $\left(\mathrm{n}^{\circ \times} 54\right.$ et 59$)$ :

Chez le premier de nos malades, cultivateur âgé de 40 ans, originaire de la province de Phu-Tho, au Tonkin, notre attention avait été attirée par l'abondance inusitée des œufs de Clonorchis sinensis dans ses selles. C'était un grand mangeur de poisson cru. Il se plaignait de douleurs abdominales et de diarrhée depuis cinq ans, d'œè̀mes généralisés depuis un an. Sa démarche était hésitante, mais ses réflexes étaient conservés. Ses urines étaient rares, non albumineuses; il toussait depuis un an. Il était très anémié. Il avait, avant la mort, trois selles diarrhéiques par jour. L'anorexie était complète, le pouls rapide ; l'état général s'aggrava rapidement et le malade mourut le 7 février 1936.

La même année, nous avons observé un second cas de distomatose pancréatique chez un individu de 39 ans, originaire de Ninh-Binh, entré 
à l'hôpital René-Robin pour diarrhée persistante (une dizaine de selles par jour). Il avait, depuis 10 jours, du subictère, des œdèmes, de l'ascite, des douleurs abdominales de plus en plus intenses, le foie douloureux. L'anorexie était complète, l'asthénie très marquée. L'anémie augmentait rapidement. Le malade mourut dans un état de cachexie extrême.

Dans les deux cas, l'infestation du foie par les douves était considérable, comme on pouvait en juger par la ditatation et la sclérose étendue des canaux hépatiques superficiels faisant saillie à la surface de l'organe, sous forme de nodules ou de cordons blanchâtres. De chaque canal sectionné s'échappait un nombre considérable de parasites, qui étaient également très abondants dans la vésicule biliaire et dans le duodénum. Dans le second cas, le foie, qui pesait 1.500 grammes, était encore plus parasité que dans le premier.

Le pancréas était de couleur jaunâtre. dur, hypertrophié, bosselé par endroits dans le cas $n^{\circ} 2$. L’infestation était faible dans le premier cas. Les douves se trouvaient au nombre de 15 environ dans le canal de Wirsung, à sa partie moyenne. Le canal était dilaté, ses parois épaissies et sclérosées.

Dans le second cas, l'infestation était beaucoup plus intense. La rate pesait 160 grammes. On trouvait des douves tout le long du canal et surtout à la queue de l'organe où elles étaient accumulées ; il y en avait aussi dans le canal de Santorini et dans tous les canaux latéraux afférents qui étaient également dilatés et sclérosés. La région de l'ampoule de Vater semblait normale. Les douves sont très nombreuses dans le canal cholédoque fortement dilaté au voisinage de l'ampoule.

\section{Etude comparative}

Notre étude porte sur deux observations de distomatose humaine à Clonorchis sinensis (déjà publiées, H. F.) et 11 observations de distomatose bovine à Eurytrema pancreaticum. Elle est essentiellement basée sur l'analyse comparative de documents histologiques.

Ces Distomes vivent exclusivement dans le système canalaire. Suivant le degré d'infestation, on les trouve seulement dans le Wirsung ou les canaux principaux, ou, au contraire, dans toutes les ramifications canalaires, même les plus périphériques.

Des œufs sont pondus vivants et retrouvés libres dans la lumière des canaux ; ceux qui pénètrent l'épaisseur des muqueuses canalaires ou les acini sont des œufs morts; ils s'entourent souvent d'un granulome macrophagique.

La parasitose intra-pancréatique se distribue donc d'une manière équivalente dans l'une et l'autre espèce, mais là s'arrête l'analogie. 
Les modalités réactionnelles du tissu pancréatique présentent des différences fondamentales.

\section{I) Infestation pancréatique chez les Bovidés.}

Les observations de distomatose pancréatique bovine se répartissent en : I) infestations numériquement faibles : 1 obs. ( $\left.{ }^{\circ} 409\right)$; II) infestations moyennes : 8 obs. $\left(\mathrm{n}^{\circ *} 398,399,402,403,404,406\right.$, $407,408)$; III) infestations intenses : 2 obs. $\left(\mathrm{n}^{\circ 8} 408,114\right)$.

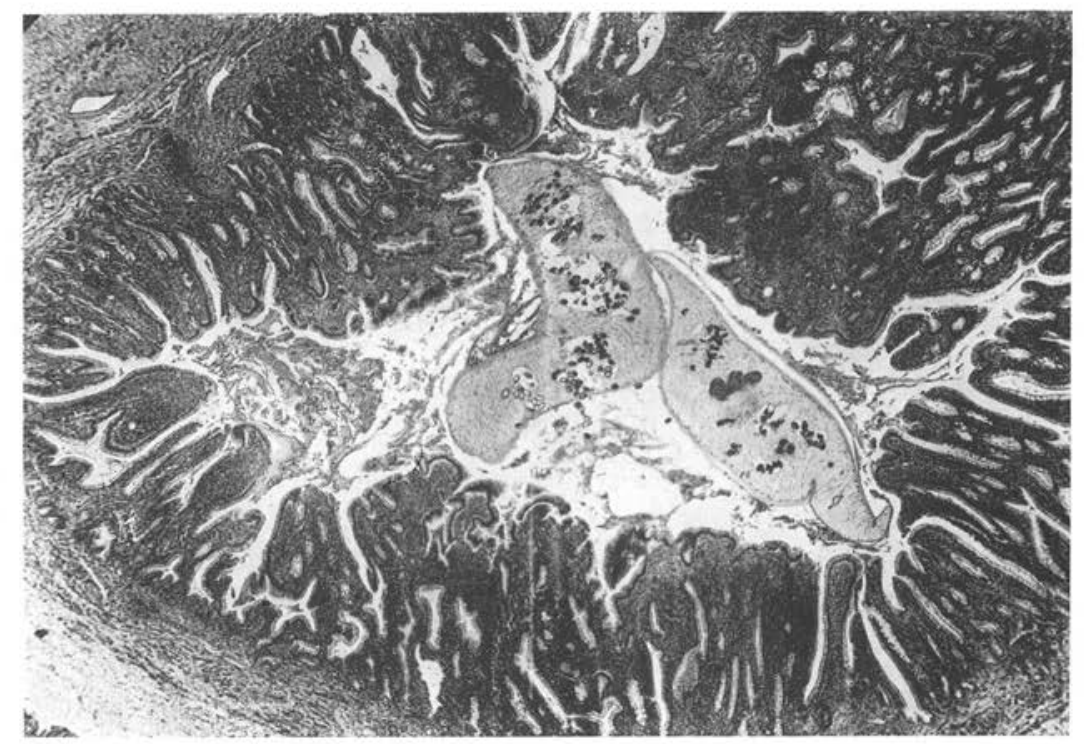

Fig. 1. - Pancréas du buffle (Eurytrema pancreaticum), obs. 398. Distension du canal principal; hyperplasie inflammatoire et métaplasie du revêtement épithélial vers le type intestinal. Dans la lumière, deux vers dont l'utérus est rempli d'œufs.

Dans le premier groupe (obs. $n^{\circ} 409$ ), il n'y a pas de vers sur la préparation, seulement quelques œufs en migration dans les voies canalaires secondaires. Le canal principal et ses affluents principaux sont de calibre normal, sans modification de leur muqueuse. L'irritation canalaire ne se manifeste, au niveau des divisions périphériques, que par une discrète sclérose en manchon et quelques foyers d'inflammation subaiguë, lymphoplasmocytaires et éosinophiliques, juxta-canalaires. Aucun retentissement parenchymateux. Les lobules acineux sont normaux, les cellules acineuses également. Les fonctions sécrétrices et excrétrices de ce tissu pancréatique apparaissent comme normales. 
Dans le deuxième groupe (infestations moyennes), on ne trouve de vers que dans le canal principal et tout au plus dans quelques-unes de ses branches principales. Suivant les préparations, on reconnait deux aspects qui correspondent peut-être à des étapes évolutives dans le temps de la parasitose :

a) Les canaux principaux contenant les vers ont subi une distension parfois considérable (obs. $\mathrm{n}^{\circ \mathrm{s}} 399,398$ ). On trouve jusqu'à cinq ou six vers libres dans la lumière. L'épithélium de revêtement est

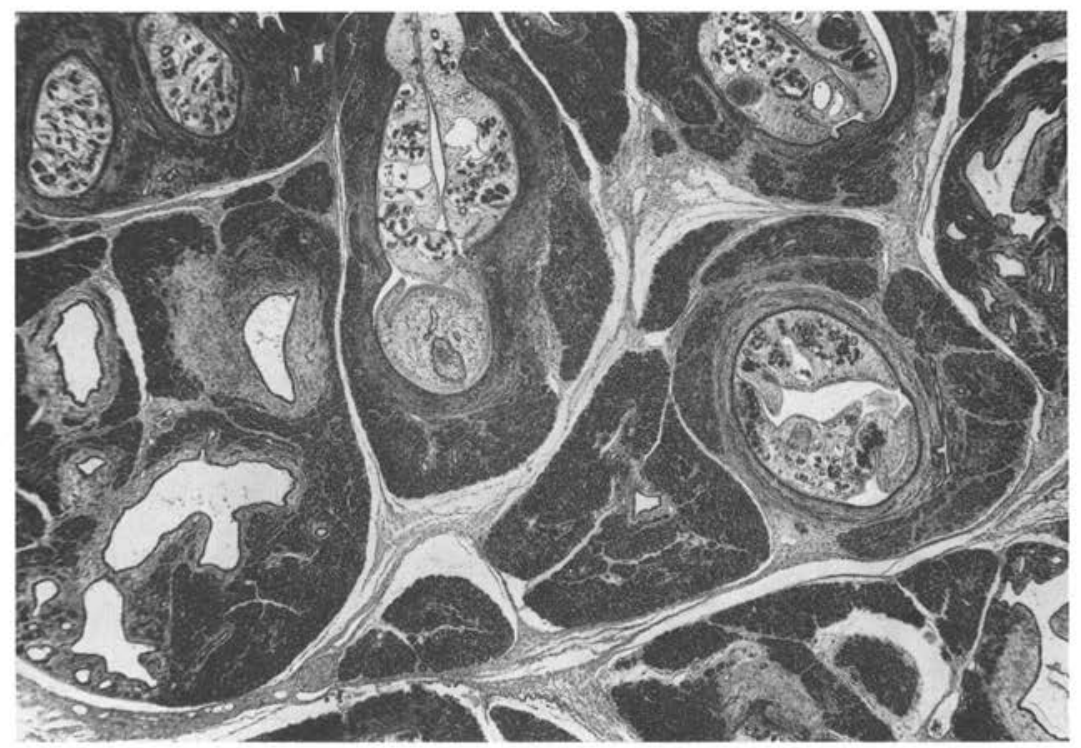

Fig. 2. - Pancréas de buflie (Eurytrema), obs. 402. Des vers cccupent la lumière de quelques gros canaux. On remarque la béance et la sclérose en manchon des canaux secondaires non parasités et l'étonnante conservation, à ce faible grossissement, d'une lobulation régulière.

hyperplasique, végétant, en même temps qu'il subit une différenciation métaplasique, qui le rapproche du type intestinal primitif (fig. 1). Cet épithélium est inflammatoire; dans le chorion, des foyers macrophagiques par corps étranger entourent des œufs morts. Une gangue scléreuse forme autour de lui un anneau épais, rigide, mais qui a l'avantage de limiter la diffusion de la réaction inflammatoire. Si les éléments acineux les plus proches présentent quelques signes de souffrance, tout le reste du tissu pancréatique, tant canaux que lobules acineux, sont normaux. 
b) Au stade suivant, les parasites sont toujours cantonnés exclusivement dans les voies canalaires principales, mais tout l'arbre canalaire est touché (obs. $\mathrm{n}^{\circ s} 402,403,404,407,405,408$ ). Les canaux sont dilatés, plus apparents que normalement parce qu'ils sont maintenus béants par une sclérose collagène en manchon très dense. Leur épithélium est soit aminci, soit au contraire hyperplasique, avec des aspects pseudo-adénomateux (fig. 2 et 3 ).

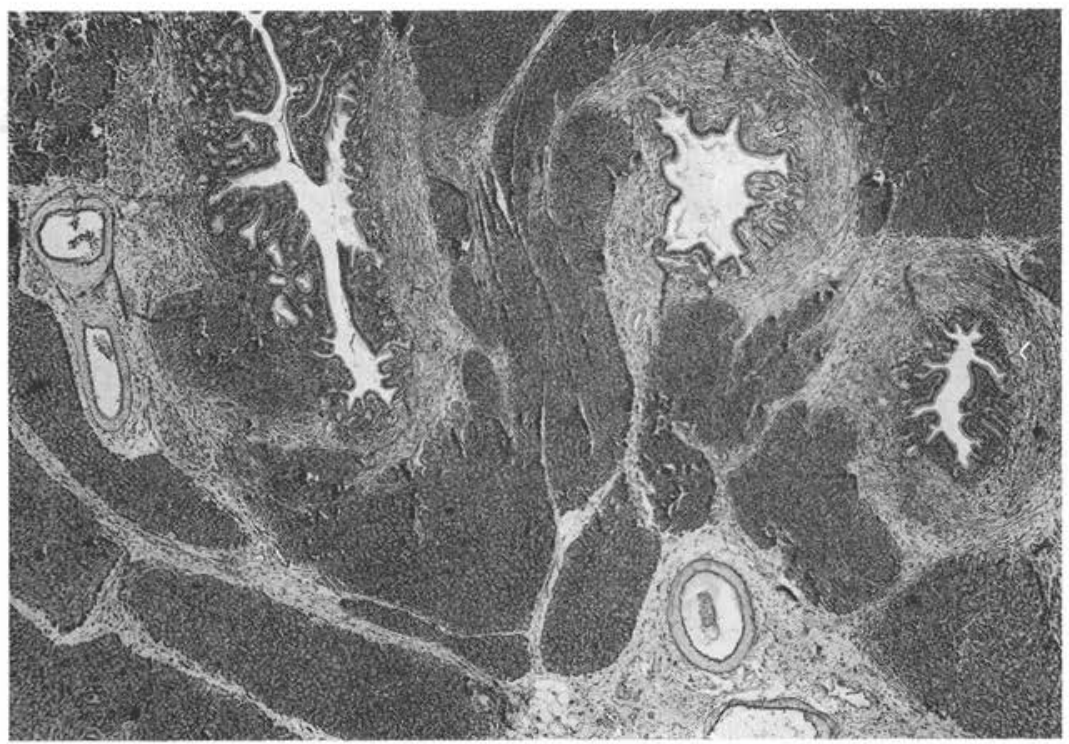

Fig. 3. - Pancréas de buffle (Eurytrema), obs. 403. A un plus fort grossissement que la photo précédente, on peut étudier des voies canalaires périphériques ne contenant pas de vers. On remarque leur sclérose adventicielle en manchon, l'hyperplasie de leur revêtement épithélial. La sclérose inflammatoire bien limitée respecte l'intégrité de la quasi-totalité du parenchyme glandulaire.

On est frappé par le peu de retentissement de cette atteinte canalaire sur le parenchyme glandulaire sécréteur. Si, au contact même des canaux dilatés, quelques acini sont étouffés et ont perdu leur rôle sécréteur, la quasi-totalité du parenchyme supporte sans dommage la parasitose et la distension canalaire pourtant considérable. Il est vrai que, d'une part, l'obstacle canalaire ne crée pas une obstruction complète, absolue ; la distension n'est pas génératrice de stase et, par conséquent, n'entraîne pas une régression canaliculaire d'amont du tissu glandulaire. D'autre part, l'inflammation des parois canalaires, rapidement sclérogène, est bien limitée. Elle ne s'ac- 
compagne d'aucune infiltration œdémateuse ou inflammatoire interstitielle dans les lobules acineux, qui conservent des fonctions normales.

Il est donc déjà possible de noter, comme étant un élément essentiel de la distomatose pancréatique des Eovidés, la systématisation canalaire et strictement canalaire de l'atteinte pancréatique ; aucun retentissement mésenchymateux ; aucun retentissement parenchymateux important.

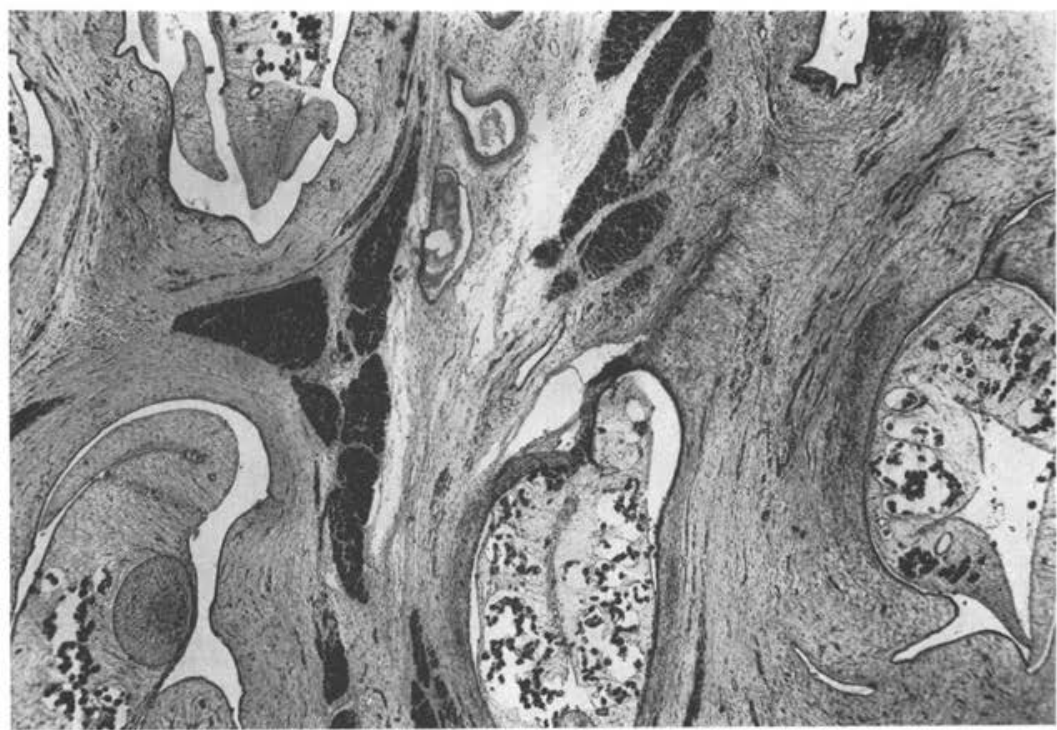

Fig. 4. - Pancréas de buffle (Eurytrema), obs. 114. Les canaux infestés sont devenus jointifs du fait de leur distension et de leur sclérose adventicielle. Les ilots acineux persistants, amenuisés, n'en sont pas moins normaux et apparemment fonctionnels.

Ces deux aspects évolutifs d'une infestation moyenne nous permettent de comprendre l'aspect véritablement extraordinaire (pour qui a l'expérience des pancréas humains) des pancréas de Bovidés parasités à l'extrême (obs. $\mathrm{n}^{\circ \approx} 114,406$ ).

On trouve des vers dans tous les canaux, en groupes ou isolés, suivant le diamètre du conduit et la taille des parasites. Quand il ne peut plus y avoir place pour un ver on trouve des œufs également groupés ou isolés. L'infestation est numériquement intense.

Les voies canalaires sont dilatées à l'extrême ; la sclérose de leur adventice est épaisse et occupe dans le lobule acineux une surface considérable. Il est certain qu'une partie des structures glandulaires 
a été étouffée, amenuisée par cette sclérose. Mais, fait essentiel, comme dans les observations précédentes, l'inflammation ne franchit que peu (et souvent même pas du tout) la barrière du manchon scléreux. Aucune manifestation inter-lobulaire, ni péri-pancréatique, à l'exception d'un état inflammatoire des ganglions de la chaîne splénique.

On arrive ainsi à l'aspect véritablement paradoxal de l'observation $\mathrm{n}^{\circ}$ 114. La distension et la sclérose de l'arbre canalaire sont

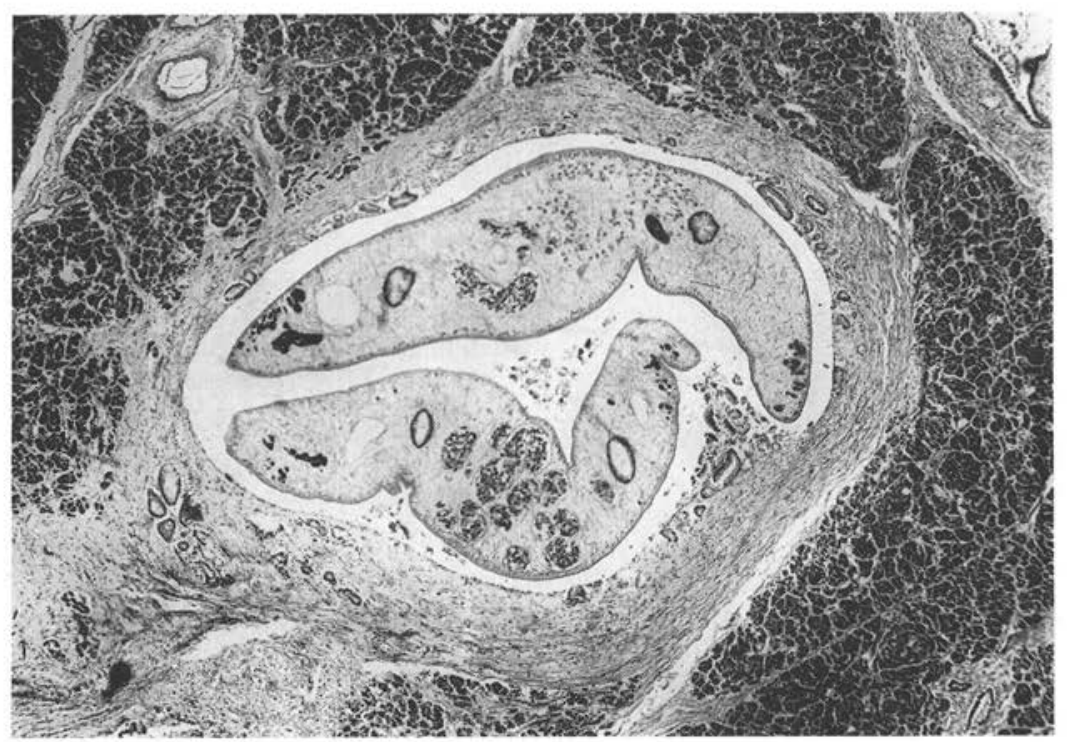

Fig. 5. - Pancréas humain (Clonorchis), obs. 54. Distension et sclérose d'un canal périphérique. Noter la sclérose inter-acineuse et même, en haut et à droite, une plage cicatricielle correspondant à la destruction de tout un lobule acineux.

telles qu'elles ne laissent persister du tissu acineux que d'étroits croissants étirés entre des canaux dont les adventices sont devenus jointifs. Mais ces petits groupes d'acini sont normalement constitués de cellules apparemment richement fonctionnelles. Leur petit nombre suffit à maintenir une sécrétion valable sur le plan vital. Quand on peut retrouver un espace inter-lobulaire, il n'est pas scléreux ; les vaisseaux et les nerfs sont normaux ; l'atmosphère péripancréatique également (fig. 4). 


\section{II) Infestation pancréatique humaine.}

Chez l'homme, la tolérance du pancréas à la parasitose est loin d'être aussi bonne que chez les Bovidés.

Nos deux observations présentent une infestation numériquement équivalente, correspondant au groupe d'infestation moyenne des Bovidés. Elles sont à des stades évolutifs différents.

a) Dans l'observation $\mathrm{n}^{\circ} 54$, les vers sont dans le Wirsung et ses branches principales. On trouve des œufs libres dans les voies cana-

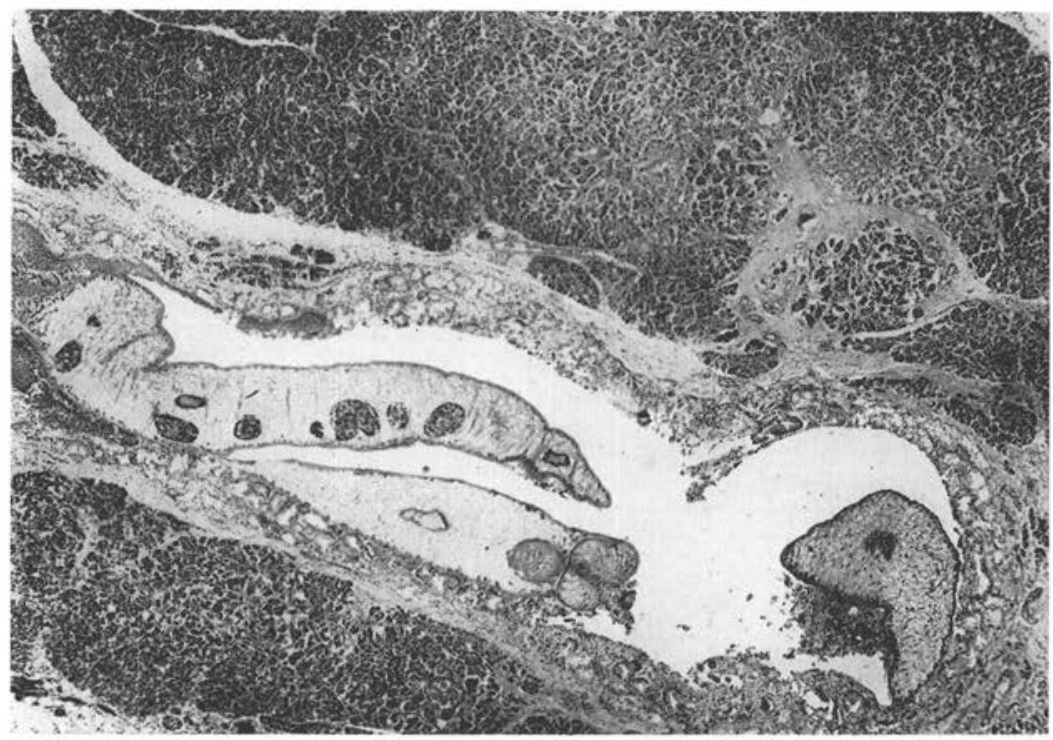

FIG. 6. - Pancréas humain (Clonorchis), obs. 59. Au contact des parasites, l'épithélium canalaire a subi une métaplasie mucoïde pseudo-acineuse; à droite l'épithélium, devenu stratifié, est de type malpighien. Importante dissociation des structures acineuses en périphérie.

laires secondaires. La distension canalaire est moyenne; elle s'accompagne, comme chez les Bovidés, d'une sclérose adventicielle importante, mais plus inflammatoire, moins nettement limitée et qui se continue en périphérie par une sclérose interstitielle systématisée des lobules.

Comme dans l'infestation animale, la parasitose ne constitue pas vraiment une oblitération complète, et, en dehors de quelques obstructions canaliculaires périphériques et segmentaires, il n'y a pas de retentissement d'amont direct. 
Les manifestations mésenchymateuses, par contre, sont considérables. La parasitose induit des poussées inflammatoires, œdémateuses, itératives, progressivement sclérogènes, chacune détruisant un peu plus l'architecture de l'organe, en laissant après elles des plages acineuses dissociées, et parfois même des petits foyers de cyto-stéato-nécrose, susceptibles de se cicatriser en formant des étoiles de tissu collagène.

Tous ces aspects se retrouvent intriqués. Les lésions pancréatiques sont manifestement en pleine évolution.

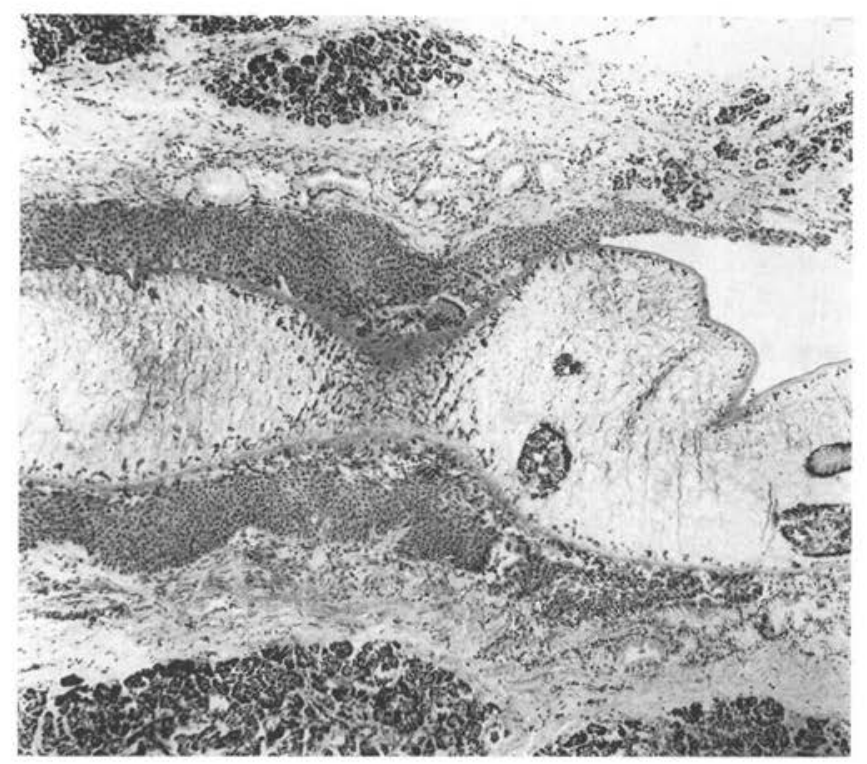

Fig. 7. - Pancréas humain (Clonorchis), obs. 59. On constate l'intensité du processus métaplasique transformant l'épithélium qui devient stratifié, du type malpighien.

L'atteinte interstitielle, inter- et intra-lobulaire, s'accompagne de remaniements inflammatoires régressifs sclérogènes de l'atmosphère péri-pancréatique, avec des lésions irréversibles des vaisseaux et des nerfs.

b) Dans l'autre observation ( $\left.\mathrm{n}^{\circ} 59\right)$, les lésions pancréatiques sont plus évoluées. C'est l'image d'une pancréatite subaiguë interstitielle grave, avec fragmentation fine et encerclement scléreux de fragments de lobules. Les groupes d'acini respectés sont dissociés par l'épaississement de leur trame de soutien et présentent des signes 
de souffrance fonctionnelle. La mutilation du pancréas est à un stade que l'on est habitué à considérer comme irréversible et susceptible d'aggravation spontanée, inéluctablement progressive, indépendamment même de la persistance de la parasitose.

On peut encore relever quelques détails lésionnels intéressants en tant que lésions élémentaires des structures pancréatiques. En plusieurs points, la présence des vers intra-canalaires a entraìné des modifications de l'épithélium de revêtement des voies excrétrices : de fréquentes dédifférenciations dans le sens intestinal ou mucoïde, avec en périphérie du canal des aspects polyadénomateux également mucoïdes ; parfois, aussi, des zones de métaplasie épidermoïde plus ou moins différenciée (fig. 5 et 6 ).

En conclusion, il apparaît done que la présence des parasites est infiniment moins bien tolérée par le pancréas humain que par celui des Povidés. Chez l'homme, indépendamment des lésions canalaires directes, la distomatose produit une pancréatite interstitielle grave, évoluant par poussées aiguës ou subaiguës vers une sclérose irréversiblement mutilante. Rapidement, l'état du pancréas est incompatible avec un fonctionnement efficace de l'organe. Dans la deuxième de nos deux olservations, le malade n'avait que trente ans.

Mais, dans l'interprétation de ces faits, et avant d'opposer dans l'absolu les modalités réactionnelles du pancréas de l'homme et des Bovidés infestés par les Distomes, il faut tenir compte du rôle que peuvent jouer certaines différences morphologiques, comme la structure spécifiquement différente du pancréas, et biologiques.

Chez le bœuf, le parenchyme pancréatique est plus finement, plus nettement segmenté, avec des voies canalaires longtemps indépentes du tissu glandulaire et mieux susceptibles de s'individualiser dans leur pathologie que celles de l'homme.

A l'opposé, chez l'homme, les voies excrétrices sont étroitement en rapport avec les structures acineuses : toute lésion canalaire retentit immédiatement sur le fonctionnement des acini.

De plus, il s'agit de deux parasites essentiellement différents dans leur comportement biologique et leur adaptation; le pancréas est l'habitat normal de l'Eurytrema pancreaticum, quelle que soit l'importance de l'infestation; chez l'homme, le Clonorchis sinensis, hôte des voies hépato-biliaires, ne pénètre le pancréas qu'exceptionnellement et au cours d'une hyper-infestation.

Chez l'homme encore, il ne faut pas oublier que, du fait de la sensibilité particulière du carrefour hépato-biliaire, le pancréas, avant 
même qu'il soit pénétré par les parasites, a pu être fragilisé par des poussées œedémateuses, du seul fait de la migration des vers dans les voies biliaires.

\section{(Institut de Parasitologie - Faculté de Médecine de Paris)}

\section{Bibliographie}

Askanazy (M.), 1904. - Die aetiologie und pathologie der katzene gerkrankung des menschen. Deutsch. med. Wochenschr., p. 689.

Fisueired (C.), Burle (De), 1928. - Sobre as lesoes do pancreas de Boi produzidas pelo Eurytrema pancreaticum. Supl. mem. inst. Oswaldo Cruz., 3.

Galliard (H.), 1936. - Un nouveau cas de distomatose pancréatique à Clonorchis sinensis. Bull. de la Soc. Médico-Chirur. de l'Indochine, $\mathrm{n}^{\circ} 5$, p. $1-2$.

Galliard (H.), Dang Van Ngu et Phan Huy Quat., 1938. - Distomatose pancréatique à Clonorchis sinensis au Tonkin. CR. du Xe Congrès de la Far East. Assoc. of Trop. med. Hanö̈, t. II, p. 659.

- Phax Huy Quat et Dang Vax $\mathrm{N}_{\mathrm{gu}}, 1936$. - Le troisième cas de distomatose pancréatique à Clonorchis sinensis observé au Tonkin. Bull. de la Soc. Méd. chirur. de l'Indcchine, $\mathrm{n}^{\circ} 4$, p. 1-5.

Hoogland (H. J. M.). - Een geval van pancreas distomatose bij de kat. Pathologisch institut van de facultelt der Veeartsenijkunde der Rijks-Universiteit te Utrecht.

Honta (P.P.), 1918. - Distomatose pancréatica e glycosuria em bovinos. A Lavoura, 22 (3e 4) : 157-8.

Katsurada, 1881. - Report on the investigation of Distomum endemicum in Okyama prefecture. Sei ni kuai fl., Tokyo, p. 151.

Looss (A.), 1914. - Wurmer und die von iihnen hervorgerufenen Erkrankungen. Mense Habd. d. Tropenkrankh. II, p. 311.

Low (R. B.), 1909. - Fasciolida in pancreas. Journ. of. Trop. Med. and Hyg., vol. XII, p. 208-209.

Magarhinos Torres (C.) et Pinto (Cesar), 1936. - Processos pathogenicos determinacos pelos trematoides Eurytrema fastosum et E. coelomaticum. Memor do Instit. Oswaldo Cruz., XXXI, p. 731.

Nencioni (C.), 1906. - Girrosi pancreatica da Distoma felineum. Contributio alla pathologia comparata del pancreas. $N$. Ercelani, II, p. 26.

Newham (H. B.), 1935. - The pathology of Clonorchis infection in the pancreas. Journ. of Trop. Med. and Hyg., XXVIII, p. 437.

Sambuc (E.), 1913. - Distomatose pancréatique. Bull. soc. méd. chir. Indochine, IV, p. 331.

et Beaujan (R.), 1913. - Un cas de cachexie aqueuse chez l'homme. Distomatose hépato-pancréatique avec syndrome pseudo-béribérique. Bull. soc. méd. chir. Indochine, p. 425.

Seyparth (C.), 1920. - Parasiten im pankreas. Zentralbl. fur Bakt, u. parasitenkunde, I Abt. Originale, LXXXV, Heft I, 27 septembre, p. 27.

Yoshida (T.), 1930. - Un nouveau cas de distomatose pancréatique à Clonorchis sinensis, in dem pankreas des Menschen. Okayama-Sgakkai-Zshi, XLII, p. 2807. 